\title{
Design Analysis of Channel Filter for Digital Down Converter in WiMAX Application
}

\author{
Priya Rana \\ ME Student, Department of Electronics \& \\ Communication Engineering, \\ National Institute of Technical Teachers' Training \\ \& Research Chandigarh, India-160019
}

\author{
Rajesh Mehra \\ Associate Professor, Department of Electronics \& \\ Communication Engineering, \\ National Institute of Technical Teachers' Training \\ \& Research Chandigarh, India-160019
}

\begin{abstract}
A channel filter has been designed for Digital Down converter (DDC), that meets the standard of WiMAX in wireless communication. WiMAX is a technology emerging in the wireless communication system, in order to enhance the broadband wireless internet access. Digital Up Converter (DUC) and Digital Down Converter (DDC) are integral part of WiMAX system, that results in efficient low cost WiMAX system. In order to fulfill the spectral requirement of bandwidth reduction, effective spectral leakage and eliminating interference from adjacent channels, in WiMAX system, DDC and DUC utilizes a channel filter for pulse shaping. Channel Filter in DDC applies pulse shaping to attenuate any out of band energy in the baseband data after decimation. The raised cosine filter and Gaussian filters are the most common pulse-shaping filters in communications systems. In present paper raised cosine filter technique and Gaussian filter is utilizes and compared for designing and analysis of channel filter using MATLAB.
\end{abstract}

\section{Keywords}

WiMAX, DDC, DUC, MATLAB, RRC filter.

\section{INTRODUCTION}

Worldwide interoperability for Microwave Access (WiMAX) technology is a rising innovation with significant potential that is ready to revolutionize the broadband wireless internet access market [1]. WiMAX is a wireless technology that provides the broadband access services that supports the low cost mobile Internet applications over large distances than the standard Wi-Fi [2]. To stay aware of essential development in the interest of wireless broadband system, new advances architecture are required, which can enhance the performance of system and improve network scalability while altogether reduces the equipment cost. Consequently with increasing cost pressures on numerous wireless equipment makers, there is a noteworthy drive to decrease both capital consumption and cost of operating expenditure attributes of the communication systems infrastructure. The noteworthy prerequisite for these systems incorporate minimal cost in terms of lesser silicon area, power consumption, processing speed, flexibility and so worth. At the point when a signal is being transmitted over a wireless medium, it may be influenced by Inter-Symbol Interference (ISI) which causes the signal to misshape. With a specific end goal to avoid this, signal scaling is performed. At the transmitter, the signal is firstly up scaled from baseband to intermediate frequency (IF) signal and then transmitted. This up scaling is performed by DUC. On the receiver side, the signal is changed once more from IF to baseband frequency. This down scaling operation is performed by a DDC [3]. Digital Up Converters (DUC) and Digital Down Converters (DDC) are very critical and essential part of a WiMAX system. These use the major resources.
Thus they result in reducing the cost and improve the performance of the WiMAX system because of their low cost design and efficient implementation.

Hence DUC and DDC assume huge part in realization of modern wireless communication system. Up sampler and down sampler are used to change the sampling rate of digital signal in DUC and DDC respectively. The structure of DUC or DDC depends upon conversion ratio required. For WIMAX systems [4] the conversion ratio is typically 8 to 10 .For DDC here, decimation factor of 8 is used. The function of DDC is to translate the frequency band of interest down the spectrum without abusing the Nyquist criterion for the message bandwidth in radio receiver so that sample rate can be down converted and thus the filter requirements and processing on the desired signal become more effortlessly realizable[5]. Filters are used in the realization of DDC for pulse shaping. Pulse shaping filters plays an important role in modern wireless communication system in order to shape the signal spectrum, avoid interference, minimizing error and increase the data transmission rate. The ideal pulse shaping filter has two properties:

i. A high stop band attenuation to decrease the interference between adjacent symbols.

ii. Minimized inter symbol interferences (ISI) without increasing the bandwidth or bit error rate [6].

Channel filter performs the function of pulse shaping in DDC. Pulse shaping filters are normally implemented as finite impulse response (FIR) digital filters].Different types of filters are there for realizing the channel filter in DDC. These filters are: Gaussian filter, raised cosine, square root raised cosine, etc. Root Raised Cosine (RRC) are used filter to do pulse shaping because its response fulfill the nyquist criterion and it has cosine curve like transition band[6].Gaussian filter give better BER performance as compared to RRC filters[7]. Hence raised cosine and Gaussian technique has been used for designing channel filters for WiMAX

\section{DIGITAL DOWN CONVERTER}

The DDC is a key component of digital radios. It performs the frequency translation necessary to convert the high input sample rates typically found at the output of an analog-todigital (A/D) converter down to lower sample rates for further and easier processing. The DDC translates one or more intermediate IF channels from a set of specified center frequencies to baseband form, forms a integral part of wireless receiver [3]. It also performs decimation and matched filtering to remove adjacent channels and maximize the received signal-to-noise ratio (SNR) [8]. A digital down converter (DDC) provides the link between the analog RF front end and the digital baseband of a receiver. The data is demodulated 
from the high frequency carrier and subsequently the sampling frequency of the data stream is reduced [1]. The reduction in the sampling rate is required to reduce the complexity of the digital system [7]. The data stream is then compatible with the baseband modem. Figurel shows a Digital down converter (DDC) for a WiMAX system.

The DDC consists of three stages shown:

1. Mixer - A numerically controlled oscillator (NCO) generates two orthogonal sinusoids at the carrier frequency and these are mixed with the input stream from the analogdigital-converter (ADC).

2. Decimation - The purpose of decimation filter is to remove all the out of band signals and noise and to reduce the sampling rate from oversampled frequency of sigma delta modulator to nyquist rate of channel [9].The sampling frequency of the intermediate frequency (IF) samples are decreased. Filtering is required to guard against aliasing in the decimation process. Decimation by the factor of 8 is down in two blocks of FIR lowpass filter. First filter will decimate the sampling rate by the factor of 4 and second filter decimate the sampling rate provided by first filter by the factor of 2 . Filters like Halfband filter, Cascaded Integrator Comb (CIC) filter, Root Raised Cosine (RRC) filter utilizes in decimation operation.

3. Channel Filter - Applies pulse shaping to attenuate any out of band energy in the baseband data. This filter requires the sharpest roll off and so has the most taps. RRC and Gaussian pulse shaping filters are used in realizing the channel filter.

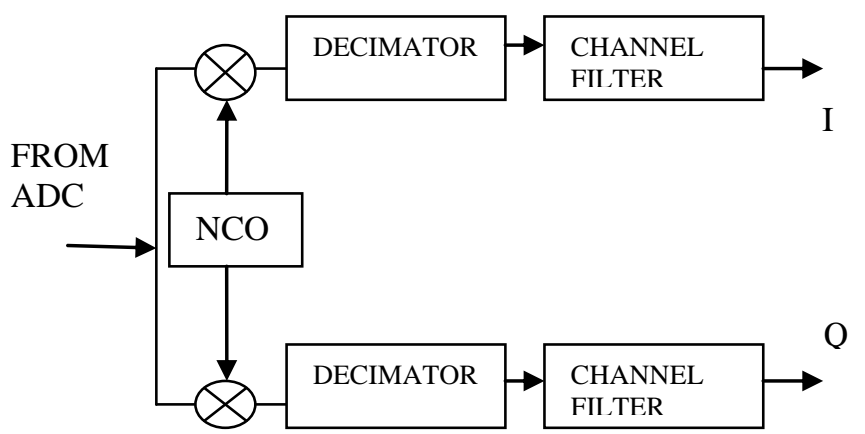

Figure1 Digital Down Converter block diagram [1]

\section{PULSE SHAPING}

In communication system, two vital prerequisites of a wireless communications channel request the utilization of pulse shaping filter. These necessities are:

1) Generate band limited channels.

2) Reduce ISI (Inter Symbol Interference).

These necessities are refined by applying pulse shaping filtering to each symbol. In order to meet the spectral requirement of wireless communication, pulse shaping plays a essential role in spectral shaping to reduce spectral bandwidth. It is a technique in spectral processing used to reduce the spectral out of band power for low cost, reliable and spectrally efficient wireless communication system. These filters along with reducing ISI also reduce adjacent channel interference [10]. In any transmission system, the transmitter send the pulses and these pulses are detected by the receiver. At receiver the received signal is sampled at an optimal point in pulse interval in order to make the probability of accurate binary decision making maximum. This suggests that no interference with one another occur at the optimal sampling point. Two criteria guarantee the non interference. Criterion one is that the pulse shape exhibits a zero crossing at the sampling point of all pulse intervals except its own. Otherwise, the residual effect of other pulses will introduce errors into the decision making process. Criterion two is that the shape of the pulses be such that the amplitude decays rapidly outside of the pulse interval [6]. When pulse shaping filter perform the function of generating signals such that each symbol period does not overlap, the matched filter is required to filter out what signal reflections do occur in the transmission process. As a direct-path signal arrives at the receiver before a reflected signal does, it is possible for the reflected signal to overlap with a subsequent symbol period. This is shown in figure 2. It is clear that, the matched filter reduces this affect by attenuating the beginning and ending of each symbol period. Thus, it is able to reduce inter symbol interference [10].

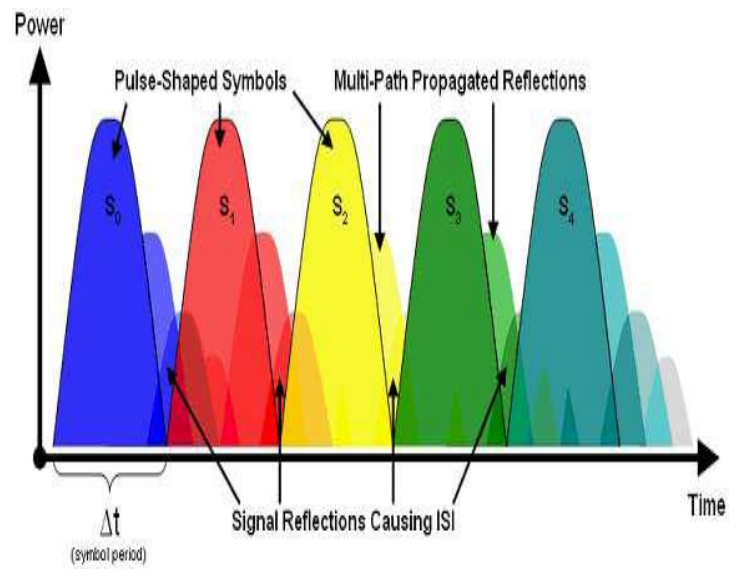

Figure2 Inter Symbol Interference (ISI)

$[10,11]$

Thus, the pulse shape utilizes in transmission should have low bandwidth and have no ISI. These two requirements are fulfilled by utilizing sinc pulse (figure 3 ) as it increase the spectral efficiency and because of windowing effect that it has on each symbol period of a modulated signal. The sinc pulse has periodic nature and its amplitude is maximum in the middle of the symbol table. Also, it is just like a square wave in the frequency domain and can limit a communication channel effectively to a specific frequency range. But it has the disadvantages of highly susceptibility to timing jitter and phase error.

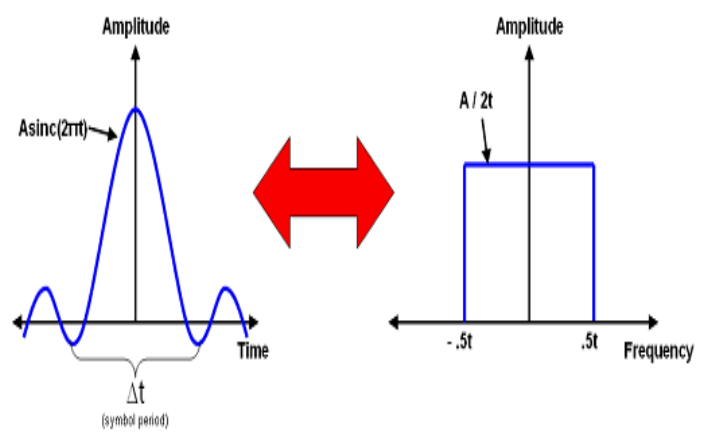

Figure3 Time and Frequency response of Sinc pulse [10, 11] 
The unbounded frequency response of the rectangular pulse renders it unsuitable for modern transmission systems. This is where pulse shaping filters come into play. Oversampled FIR digital filters are preferred more for implementing the pulse shaping filters because of their advantages of stability, linear phase and non feedback structure. The FIR structure with linear phase technique is efficient as it takes advantage of half the required multiplications and addition [11]. FIR Gaussian pulse shaping filter and RRC (Root raised cosine) pulse shaping filters are employed in the channel filter of DDC for pulse shaping purpose. Gaussian filters are better than RRC filters as they give better BER performance than RRC filters [11].

\subsection{Gaussian Pulse Shaping Filter}

Gaussian pulse shaping filter is a filter having Gaussian function as its impulse response. Gaussian filter give no overshoot to a input step function and also reduces the rise and fall time. Because of its linear property it is used as smoother. It exhibit minimal group delay. Modification of the input signal by Gaussian filter is done by performing convolution operation between input signal and Gaussian function. The FIR Gaussian pulse-shaping filter design is done by truncating a sampled version of the continuous-time impulse response of the Gaussian filter which is given by:

$$
h(t)=\left(\frac{\sqrt{\pi}}{a}\right) e^{-\frac{\pi^{2} t^{2}}{a^{2}}}
$$

The parameter ' $a$ ' is related to 3-dB bandwidth-symbol time product $(\mathrm{B} * \mathrm{Ts})$ of the Gaussian filter as given by:

$$
\mathrm{a}=\frac{1}{B T_{S}} \sqrt{\frac{\log 2}{2}}
$$

There are two approximation errors in the design: a truncation error and a sampling error. The truncation error is due to a finite-time (FIR) approximation of the theoretically infinite impulse response of the ideal Gaussian filter. The sampling error (aliasing) is due to the fact that a Gaussian frequency response is not really band-limited in a strict sense (i.e. the energy of the Gaussian signal beyond a certain frequency is not exactly zero). This can be noted from the transfer function of the continuous-time Gaussian filter, which is given as below:

$$
\mathrm{H}(\mathrm{f})=e^{-a^{2} f^{2}}
$$

As $f$ increases, the frequency response tends to zero, but never is exactly zero, which means that it cannot be sampled without some aliasing occurring.

Graphical representation of the impulse response of Gaussian filter is shown in figure4. It is clear that there are no zero crossings for this type of filter.

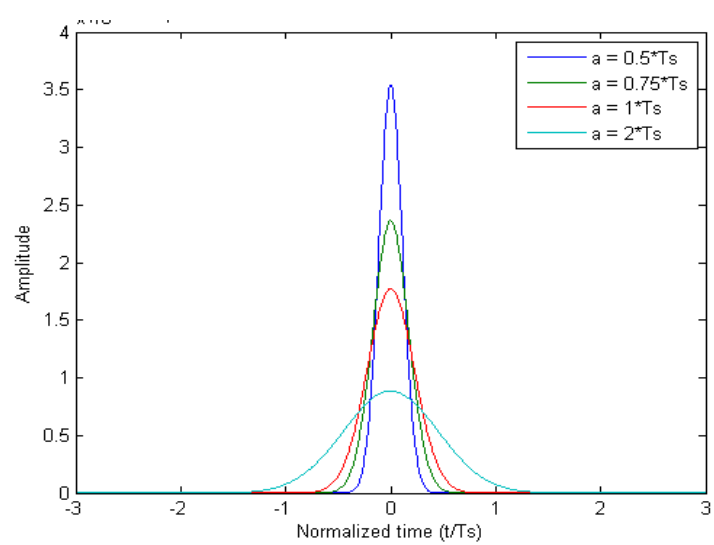

Figure4 Impulse Response of Gaussian Filter

\subsection{Raised Cosine Pulse Shaping Filter}

In numerous data transmission application, the transmitter signal must be confined to certain bandwidth. This can be due to system design constraints. In such examples, the infinite bandwidth associate with rectangular pulses is not adequate. The bandwidth of rectangular pulses is not adequate. The bandwidth of rectangular pulse can be constrained, in any case, by compelling to pass throw a low pass filter. This filtering results in change of shape of rectangular pulse to smooth from without sharp edges. Hence, filtering rectangular data pulses is often referred to as pulse shaping. By limiting the bandwidth of rectangular pulse introduces a damped oscillation i.e. the rectangular pulses exhibit non zero amplitude only during the pulse interval where as the filtered pulse consist ripples both before and after the pulse interval. At the receiver, because of interference of ripples associated with one pulse with pulses before and after it, results in incorrect decoding of data. Hence, selection of proper filter can give the desired bandwidth reduction while maintaining a time domain shape [10]. This filter is raised cosine filter and its frequency response is

$$
\begin{array}{cl}
\mathrm{H}(\mathrm{w})=\tau & 0 \leq \mathrm{w} \leq \mathrm{c} \\
\tau\{\cos 2[\tau(\mathrm{w}-\mathrm{c}) / 4 \beta]\} & \mathrm{c} \leq \mathrm{w} \leq \mathrm{d} \\
0 & \mathrm{w}>\mathrm{d}
\end{array}
$$

where $\mathrm{w}$ is the radian frequency $2 \pi \mathrm{f}$,

$\tau$ is the pulse period

$\beta$ is roll off factor

$\mathrm{c}$ is equal to $\pi(1-\alpha) / \tau$

$\mathrm{d}$ is equal to $\pi(1+\alpha) / \tau$

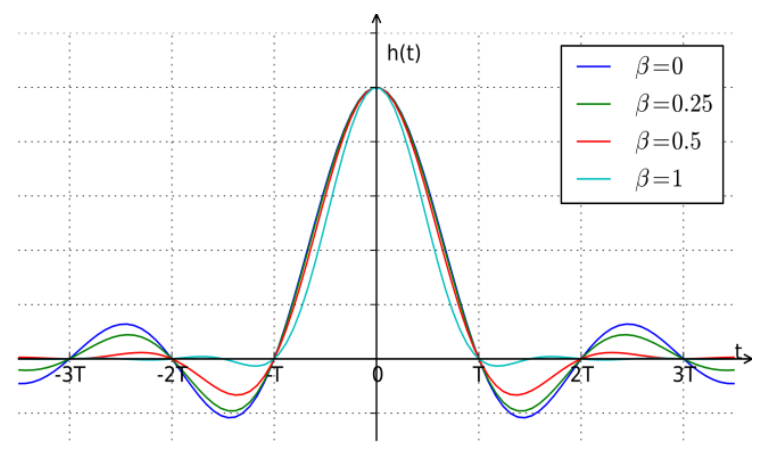

Figure5 Impulse response of Raised Cosine Filter 
Root raised cosine (RRC) is widely used as a matching filter in transmission and receiving sections in digital communication system to reduce the ISI. This filter is characterized by roll off factor $(\beta)$ and the reciprocal of the symbol rate. For pulse shaping in DDC and DUC, RRC filters are preferred [5].

\section{CHANNEL FILTER DESIGN}

Channel filters for DDC for WiMAX applications are designed using Gaussian pulse shaping and raised cosine pulse shaping FIR filters in MATLAB using fdatool that satisfy the WiMAX requirements. The sampling frequency for the channel filter in WiMAX is $11.424 \mathrm{Msps}$. Number of taps $=111$. Thus, filter order is $=111-1=110$.cutt off frequency $=$ 4Msps for both Gaussian and raised cosine pulse shaping technique.

The FIR Gaussian pulse shaping filter design is done by truncating a sampled version of continuous time impulse response of Gaussian filters. The proposed Gaussian filter has been designed and simulated using MATLAB.

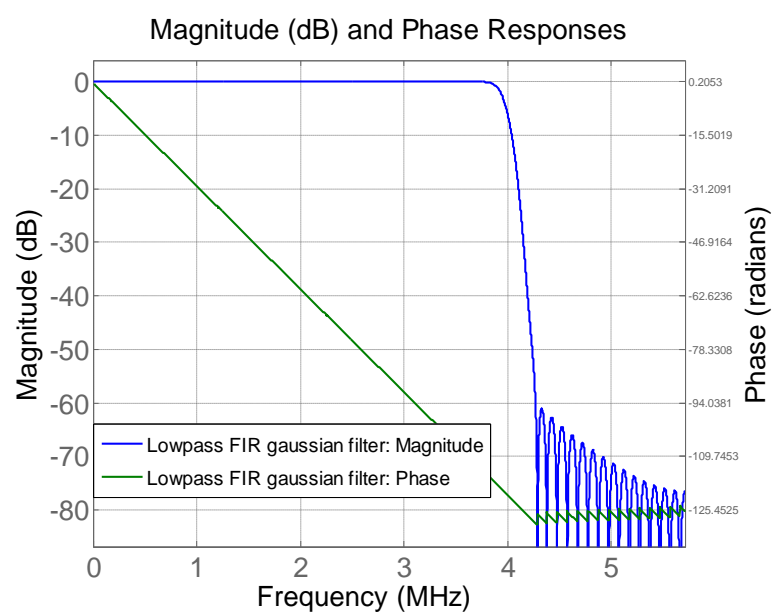

Figure6 WiMAX Gaussian filter for WiMAX

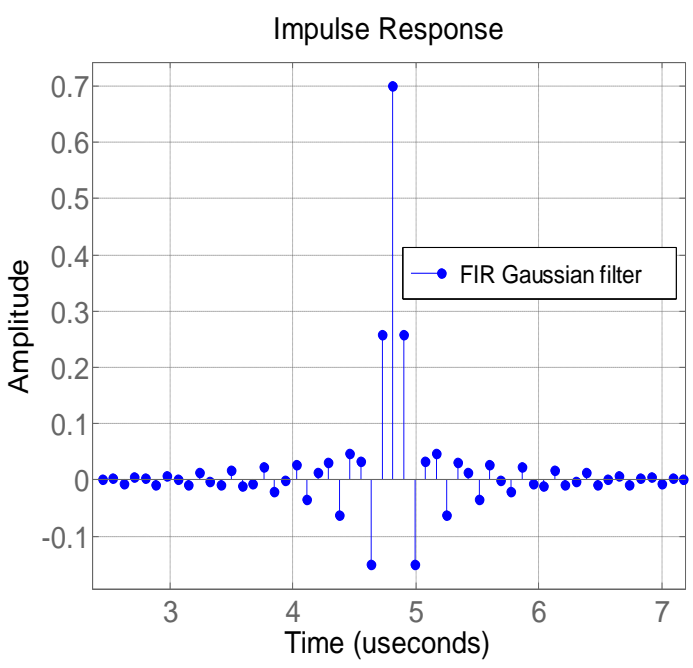

Figure7 Impulse response of Gaussian channel filter

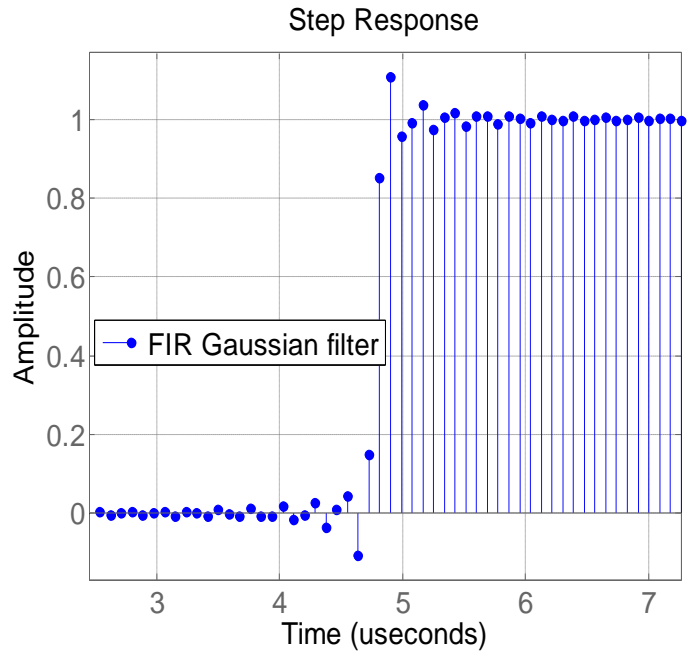

Figure8 Step response of Gaussian channel filter

The raised cosine filter is obtained by truncating the analytical impulse response and is not optimal because it results in higher filter order. In this proposed work first raised cosine filter is designed using filter order 110 , roll off factor .25 and using hanning window.

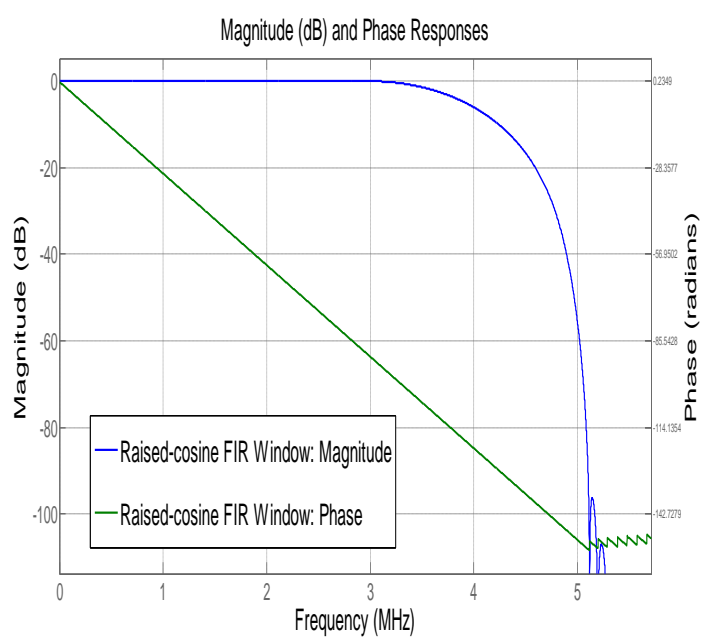

Figure9 Raised Cosine channel filter for WiMAX

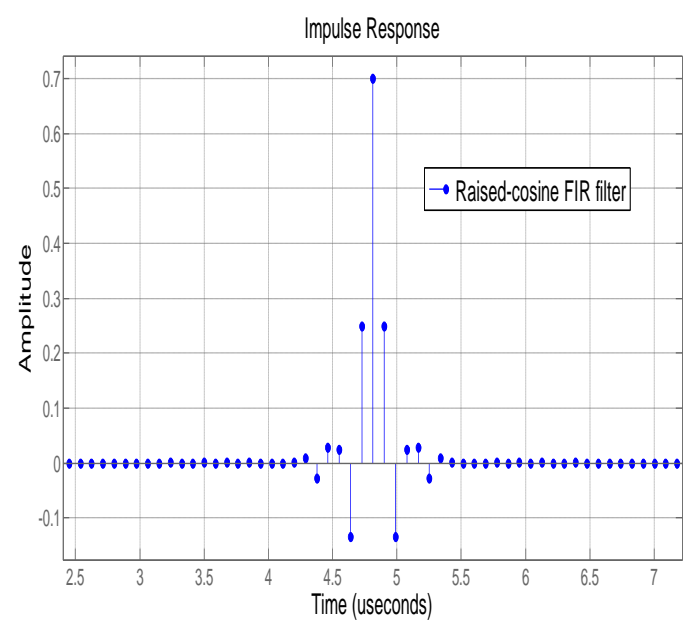

Figure10 Impulse response of Raised cosine channel filter 


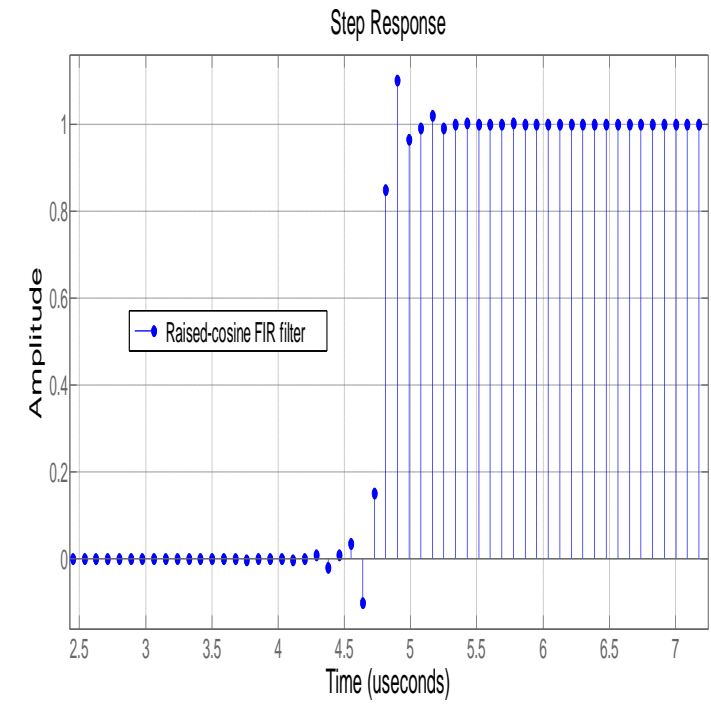

Figure11 Step response of Raised cosine channel filter

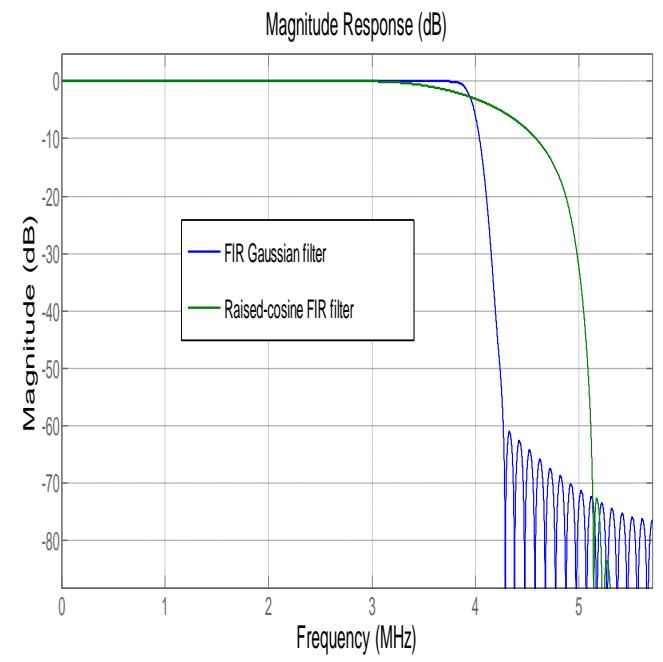

Figure12 Performance comparison

\section{COMPARATIVE ANALYSIS}

The performance and cost of both the pulse shaping filters have been analyzed and compared. It is cleared from figure 6 and 8 , that raised cosine filter provide large transition width and low stopband attenuation as compared to the Gaussian filter in magnitude response. Phase response of the Gaussian filter is more linear in passband as compared to RRC filter.

Table1 Implementation cost comparison

\begin{tabular}{|c|l|c|c|}
\hline $\mathbf{1}$ & TECHNIQUE & $\begin{array}{c}\text { Gaussian } \\
\text { Filter }\end{array}$ & $\begin{array}{c}\text { Root } \\
\text { Raised } \\
\text { Cosine } \\
\text { Filter }\end{array}$ \\
\hline $\mathbf{2}$ & $\begin{array}{l}\text { NO. OF } \\
\text { MULTIPLIERS }\end{array}$ & 111 & 109 \\
\hline $\mathbf{3}$ & NO. OF ADDER & 110 & 108 \\
\hline $\mathbf{4}$ & NO. OF STATES & 110 & 110 \\
\hline $\mathbf{5}$ & $\begin{array}{l}\text { MULTIPLICATION } \\
\text { PER INPUT } \\
\text { SAMPLE }\end{array}$ & 111 & 109 \\
\hline $\mathbf{6}$ & $\begin{array}{l}\text { ADDITION PER } \\
\text { INPUT SAMPLE }\end{array}$ & 110 & 108 \\
\hline
\end{tabular}

From the table, it is cleared that the Gaussian filters require more no. of multiplies, adders and multiplication and additions per input sample, as compared to the root raised cosine filter. Hence Gaussian filters have more implementation cost in term of hardware requirement.

\section{CONCLUSION}

In this paper, digital filter for DDC in WiMAX application is designed using Gaussian and RRC pulse shaping filters in order to meet the spectral requirement of wireless communication. Filters designed by using two techniques are then compared. It is concluded that because of small transition bandwidth and more linear phase in magnitude and phase response of Gaussian filter as compared to RRC filter, Gaussian filter give significantly better BER performance than conventional RRC filter. But Gaussian filter has disadvantages of large stopband attenuation, and more implementation cost of hardware requirements, hence RRC filter is preferred in applications where cost is major factor. In WiMAX for utilizing channel filter in DDC, RRC filter is more preferred. The future scope of this channel filter is that it can be used as in 5G and other advance generations for pulse shaping and noise filtering.

\section{ACKNOWLEDGEMENTS}

The author would like to thank Director and Head of Electronics and Communication Engineering Department, National Institute of Technical Teachers' Training \& Research, Chandigarh, India and for their constant inspirations, support and helpful suggestions throughout this research work.

\section{REFERENCES}

[1] Altera "Accelerating DUC and DDC system designs for WiMAX" Version 2.2, May 2007.

[2] Deepika Rathore, Rajeev Paulus, A.K. Jaiswal, Mayur Kumar "Performance evaluation of a WiMAX mobile network using omnidirectional antennas" International Journal of Current Engineering and Technology, Volume .4, Issue No.2, Page no.909-912, April 12014.

[3] Shrita G, A.R Aswatha "Design and Implementation of an Efficient 4G Digital down Converter used in Wireless Receiver Systems" International Journal of Computer Applications (0975 - 8887) Volume 120, Issue No.18, Page no.17-20, June 2015.

[4] Sumeet Prashar, Rajesh Mehra "Design and Analysis of Multirate Filter for WiMAX Application" International Journal of Engineering Research and Development Volume 5, Issue no. 2, Page no.60-63, December 2012.

[5] Ravi Kishore Kodali, Seeta Rami Reddy Kondapalli, Dr. Lakshmi Boppana "DDC and DUC Filters in SDR platforms" Conference on Advances in Communication and Control Systems 2013 (CAC2S 2013), Page no.203208, 2013.

[6] Rajesh Mehra and Swapna Devi "Area Efficient \& Cost Effective Pulse Shaping Filter For Software Radios" International Journal of Ad hoc, Sensor \& Ubiquitous Computing (IJASUC) Volume 1, Issue no.3, Page no.8591, September 2010.

[7] Rajesh Mehra, Ginne "Area Estimation and Design Analysis of Gaussian Pulse Shaping Filter" International Journal of Soft Computing and Engineering(IJSCE),Volume 3, Issue no. 3, Page no.211214, July 2013 
[8] Stephen Creaney and Igor Kostarnov "Designing Efficient Digital Up and Down Converters for Narrowband Systems" XilinxXAPP1113 (v1.0) November 21, 2008.

[9] Pratibha Paliwal, Manpreet Singh "Design Implementation of Digital Down Converter for WiMAX System" International Journal of Advanced Research in Computer and Communication Engineering" Volume 2, Issue 9, Page no.3336-3338, September 2013.
[10] A. S Kang, Vishal Sharma "Pulse Shape Filtering in Wireless Communication-A Critical Analysis" (IJACSA) International Journal of Advanced Computer Science and Applications, Volume 2, Issue no.3, March 2011.

[11] Rajesh Mehra, Ginne "FPGA Based Gaussian Pulse Shaping Filter Using Distributed Arithmetic Algorithm" International Journal of Scientific \& Engineering Research, Volume 4, Issue no. 8, Page no.711-715, August-2013. 